\title{
CORRECTION
}

\section{Correction to: A priori dietary patterns and blood pressure in the EPIC Florence cohort: a cross-sectional study}

\author{
B. Bendinelli ${ }^{1}$-G. Masala ${ }^{1}$ (D) R. M. Bruno ${ }^{2} \cdot$ S. Caini ${ }^{1} \cdot$ C. Saieva ${ }^{1} \cdot$ A. Boninsegni ${ }^{1} \cdot$ A. Ungar ${ }^{3,4} \cdot$ L. Ghiadoni $^{2} \cdot$ D. Palli $^{1}$
}

Published online: 10 October 2018

○) Springer-Verlag GmbH Germany, part of Springer Nature 2018

\section{Correction to: European Journal of Nutrition}

https://doi.org/10.1007/s00394-018-1758-2

In the original publication of the article Tables 3 and 4 have been published in an incorrect form. The corrected Tables 3 and 4 are given below.

The original article can be found online at https://doi.org/10.1007/ s00394-018-1758-2.

G. Masala

g.masala@ispro.toscana.it

1 Cancer Risk Factors and Lifestyle Epidemiology Unit, Institute for cancer research, prevention and clinical network, ISPRO, Via delle Oblate 4, 50141 Florence, Italy

2 Department of Clinical and Experimental Medicine, University of Pisa, Pisa, Italy

3 Division of Geriatric Cardiology and Medicine, University of Florence, Florence, Italy

4 Azienda Ospedaliero-Universitaria Careggi, Florence, Italy 


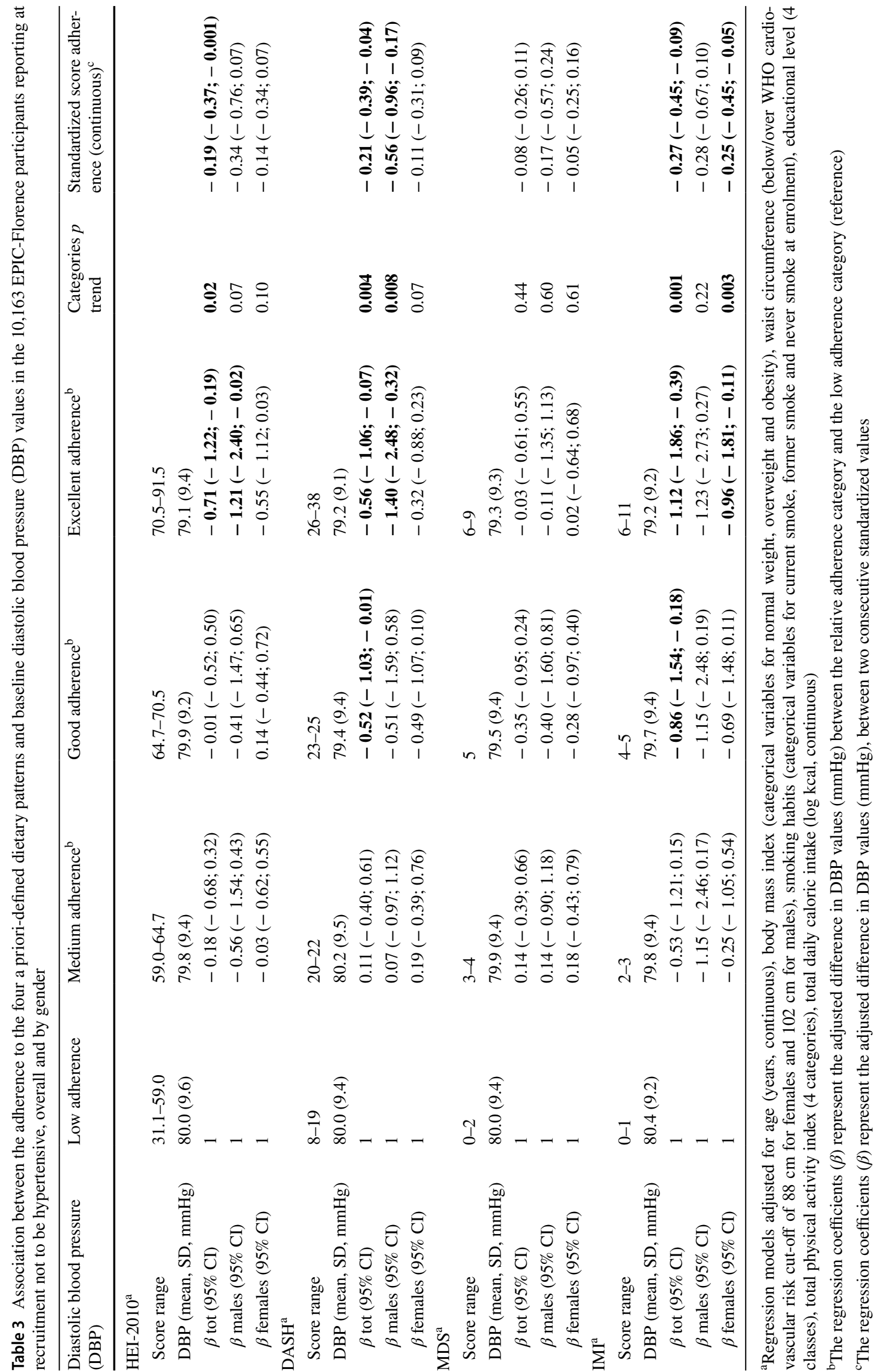




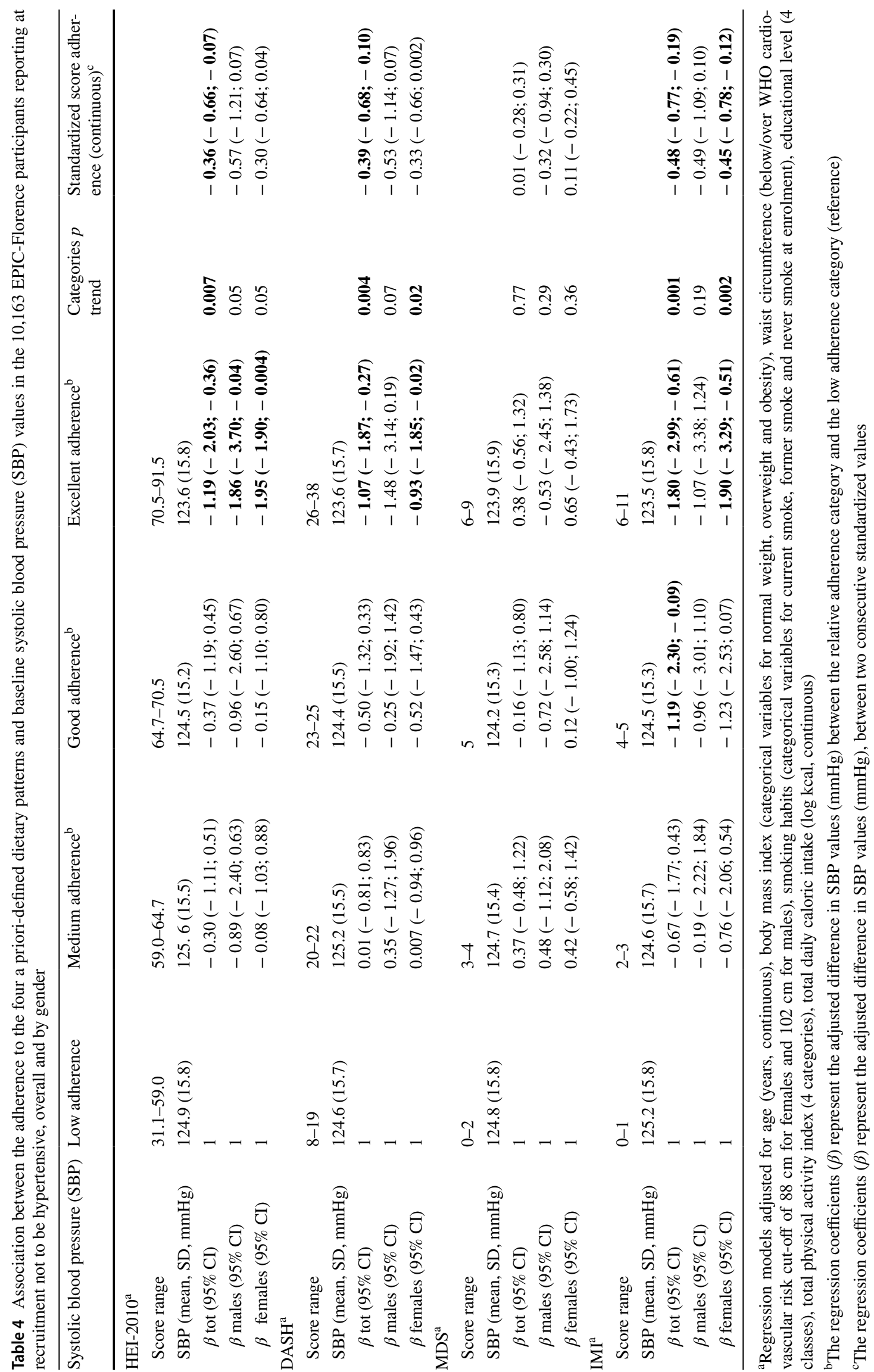

